\title{
Multiplication Technique in Residue Number System
}

\author{
Tukur Gupta, Shamim Akhter, Shaheen Khan, Saurabh Chaturvedi
}



\begin{abstract}
This paper presents the modulo multiplication technique in residue number system (RNS) using Vedic mathematics. Residue number system supports fast mathematsical computation. In this paper, the use of the combination of RNS and Vedic mathematics has improved the computation time for modulo multiplication operation. The proposed modulo multiplier is implemented in VHDL and synthesized using Xilinx ISE 14.1.The performance comparison analysis in terms of area, power and delay is done between the proposed technique and direct computation. The performance of the multiplier circuit has been compared using the $32 \mathrm{~nm}$ standard cells available in Synopsys Design Compiler. The presented Vedic modulo multiplier is efficient in terms of speed for large input data sizes.
\end{abstract}

Index Terms: HDL design, Vedic mathematics, residue number system (RNS), lookup table (LUT), modular multiplication

\section{INTRODUCTION}

High-speed multiplier is necessity in all multiplication operation, including digital signal processing, image processing and others. Therefore, a lot of focus has been put on the development of area efficient and fast multipliers. Vedic multiplier [1], [2] is considered as one of the most efficient binary multipliers because of its modular approach. The architecture of a Vedic multiplier is based on the vertical and crosswise algorithm of the ancient Indian Vedic mathematics.Residue number system (RNS) [3] is a non-weighted number system. The parallel nature for the mathematical operations in RNS results in a faster computation. In RNS, an integer number is represented as a set of residues, and these residues are processed separately. In this paper, the Vedic multiplication technique proposed in [2] has been applied on RNS, which avoided the requirement of larger sized multiplier units. The organization of this paper is as follows: Section II presents the analysis and design for the Vedic-based multiplication operation in RNS domain. Section III deals with the design strategies for different moduli set. Section IV compares the proposed modular multiplication technique with the direct computation method. Finally, the conclusions are drawn in Section V.

Revised Manuscript Received on October 30, 2019.

* Correspondence Author

Tukur Gupta*, Ph.D. Scholar, Department of Electronics and Communication Engineering (ECE), Jaypee Institute of Information Technology, NOIDA, India

Shamim Akhter, Assistant Professor, Department of ECE, Jaypee Institute of Information Technology, NOIDA, India

Shaheen Khan, Assistant Professor, Department of ECE, Mewat Engineering College (Waqf), Haryana, India

Saurabh Chaturvedi, Assistant Professor, Department of ECE, Jaypee Institute of Information Technology, NOIDA, India

(C) The Authors. Published by Blue Eyes Intelligence Engineering and Sciences Publication (BEIESP). This is an open access article under the CC BY-NC-ND license (http://creativecommons.org/licenses/by-nc-nd/4.0/)

\section{VEDIC-BASED MULTIPLICATION IN RNS}

Modulo multiplication of two inputs $\mathrm{X}$ and $\mathrm{Y}$ in RNS is represented by $|\mathrm{X} . \mathrm{Y}|_{\mathrm{m}}$, where $\mathrm{m}$ is the modulo number. Direct computation requires a multiplier to compute X.Y followed by mod-m operation to obtain the final result. The implementation of a multiplier circuit demands a large hardware in terms of logic gates. The computation of mod-m requires a lookup table (LUT), where the address size of an LUT is related to the number of bits in the input data. As an example, for 8-bit multiplication, an LUT requires 16-bit address. The LUT content width is decided by the value of moduli set $\mathrm{m}$. The requirements of multiplier and respective LUT size for direct modulo multiplication are given in Table I.

Table I. Multiplier and LUT requirements for $\mathbf{m}=7$

\begin{tabular}{|c|c|c|}
\hline $\begin{array}{c}\text { No. of input bits } \\
(\mathrm{N})\end{array}$ & $\begin{array}{c}\text { Multiplier size } \\
\text { (bits) }\end{array}$ & $\begin{array}{c}\text { LUT address size } \\
\text { (bits) }\end{array}$ \\
\hline 4 & $4 \times 4$ & 8 \\
\hline 8 & $8 \times 8$ & 16 \\
\hline 16 & $16 \times 16$ & 32 \\
\hline 32 & $32 \times 32$ & 64 \\
\hline
\end{tabular}

In this paper, the multiplication based on Vedic mathematics is proposed to reduce the requirements of multiplier and LUT. In the proposed approach, the input bits are divided into two equal parts, i.e. for $\mathrm{N} \times \mathrm{N}$ multiplication, divide the multiplicand and multiplier into two parts, consisting of ( $\mathrm{N}$ to $\mathrm{N} / 2+1)$ bits and (N/2 to 1 ) bits. As shown in Fig. 1, input data $X$ and $Y$ are divided as $X_{M} X_{L}$ and $Y_{M} Y_{L}$. Thereafter, mod-m is computed for all the four subdivided parts followed by Vedic-based cross product computation.

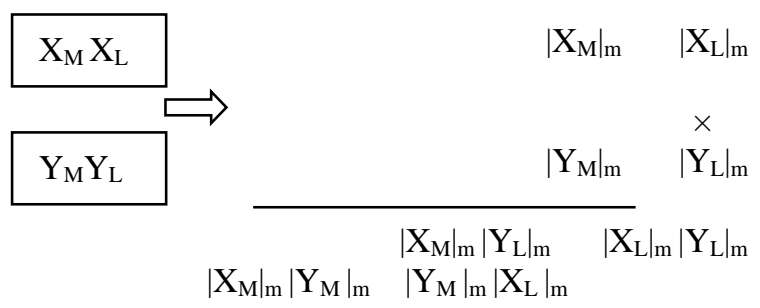

Fig. 1. Vedic-Based Cross Product Computation.

For an example, with $m=7$, the modulo of an input can be represented using maximum of 3 bits. As depicted in Fig. 1, with $\mathrm{m}=7$, the subdivided parts are multiplied using a small sized multiplier because each input to the multiplier is of 3 bits. 
The complete block diagram of the proposed architecture is demonstrated in Fig. 2 for $m=7$. The partial products after Vedic multiplication followed by mod-7 are shown as: $\mathrm{P}_{3}, \mathrm{P}_{2}$, $\mathrm{P}_{1}$ and $\mathrm{P}_{0}$. The value of $\mathrm{P}_{1}$ and $\mathrm{P}_{2}$ is shifted by 1-bit and that of $\mathrm{P}_{3}$ by 2-bit. This is because of the fact that the divided subparts are multiples of 16 , therefore a factor of 2 is taken to compensate for the factor of $|16|_{7}$, i.e. 2 , for $P_{1}$ and $P_{2}$. Similarly, for $\mathrm{P}_{3}$, a factor of 4 is taken to compensate for $|256|_{7}$, i.e. 4.

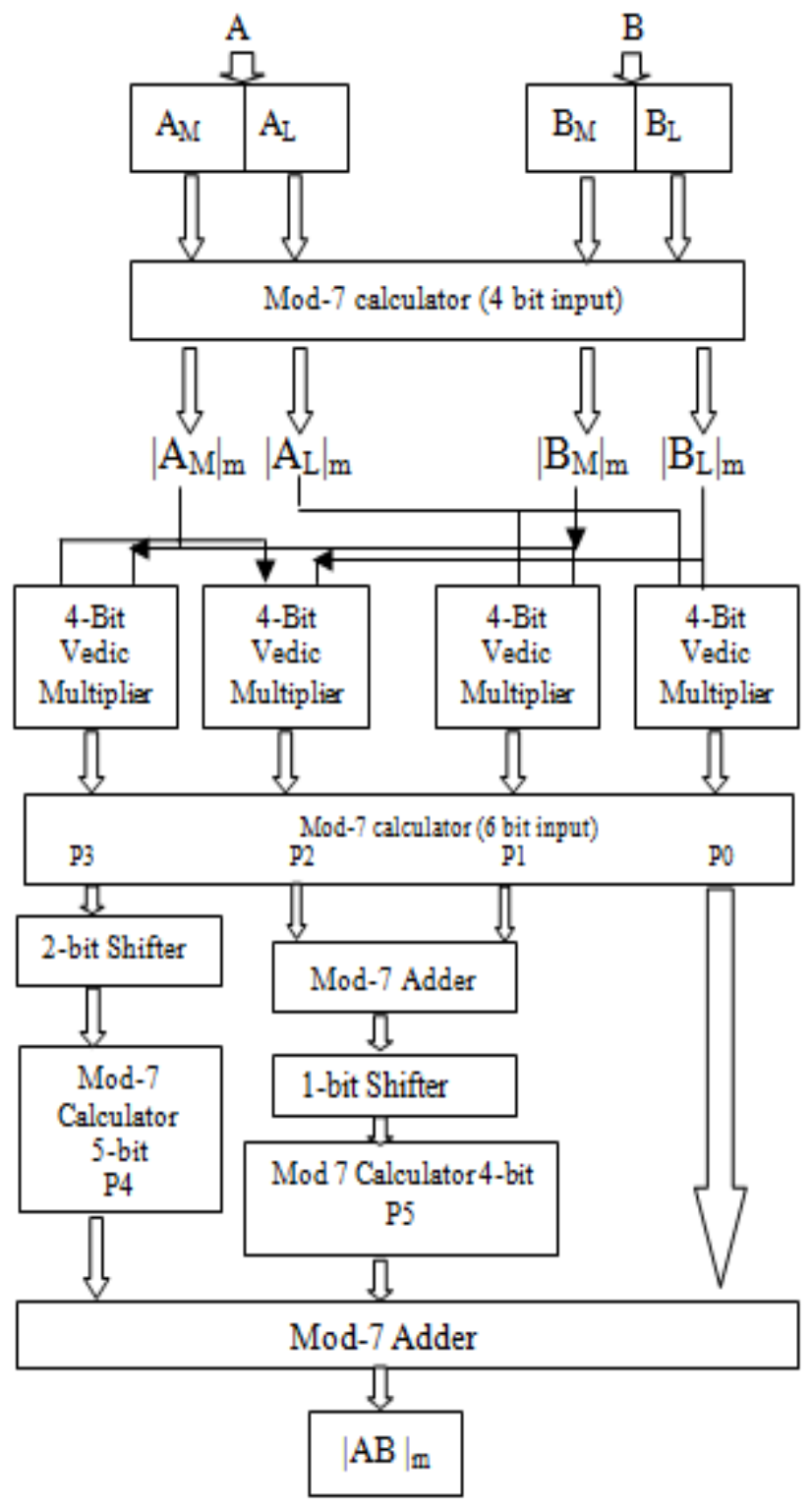

Fig. 2. Proposed RNS domain Multiplier for $\mathbf{m}=7$.

As an example, if input $\mathrm{A}=10111111$, then subdivided parts will be: $A_{M}=1011$ and $A_{L}=1111$. Similarly, if $\mathrm{B}=11101001$, then $\mathrm{B}_{\mathrm{M}}=1110$ and $\mathrm{B}_{\mathrm{L}}=1001$. These values are given to mod-7 calculator (in this case $\mathrm{m}=7$ ) as inputs.

Fig. 3 displays the architecture of the mod-7 calculator [3], and it can be extended to any modulo number with a modification in LUT size, contents and modulo adder.

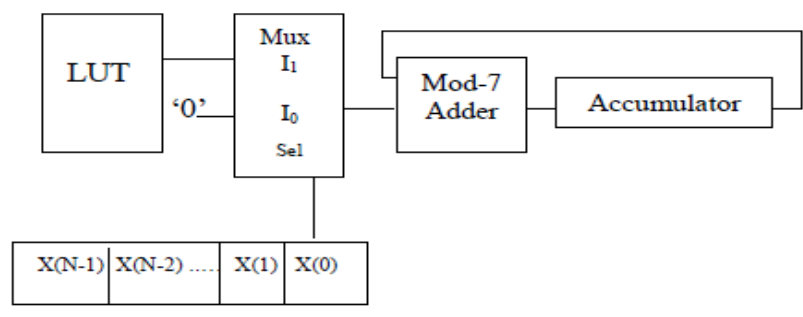

Fig. 3. Mod-7 calculator [3]

For 8-bit modulo multiplication, the subdivided parts of A and B are of 4-bit each, therefore LUT has only four address locations. The LUT contents for $\mathrm{m}=7$ are listed in Table II.

Table II. LUT Contents For Mod-7 For 4-Bit Input Data

\begin{tabular}{|c|c|}
\hline LUT address & LUT contents \\
\hline 00 & 001 \\
\hline 01 & 010 \\
\hline 10 & 100 \\
\hline 11 & 001 \\
\hline
\end{tabular}

As discussed in [3] and shown in Fig. 3, the LUT contents are derived based on value of $\left|2^{j}\right| \mathrm{m}$, where $\mathrm{j}$ varies from 0 to 3 for $m=7$. The value of input data sequence selects either the LUT content or ' 0 ' using a $2 \times 1$ multiplexer. For an example, consider the computation of $\left|\mathrm{B}_{\mathrm{L}}\right|_{7}$ with $\mathrm{B}_{\mathrm{L}}=1001$, the sequence of data out from the multiplexer will be:

$$
001=>000=>000=>001
$$

The extracted value from the multiplexer is recursively used in the modulo-7 adder [3]. The modulo-m adder produces $|A+B|_{m}$. For this example, the result of iteration is given below, with an assumption that the accumulator is initially clear.

$$
\begin{aligned}
& |000+001|_{7}=>001 \\
& |001+000|_{7}=>001 \\
& |001+000|_{7}=>001 \\
& |001+001|_{7}=>010
\end{aligned}
$$

The final result is 010 which is 2 in decimal, and it represents the value of $\left|B_{L}\right| 7$, i.e. $|9|_{7}=2$. Various architectures of modulo-m calculator and adder are presented in [4]-[7]. The block diagram of a 4-bit modified Vedic multiplier is discussed in [2] and shown in Fig. 4 with the inputs A and B. This architecture requires four 2-bit binary multipliers, a carry save adder (CSA), an OR gate and a 2-bit increment-by-one (IBO) circuit. The outputs from the 2-bit multiplier are indexed from $\mathrm{P}_{15}$ to $\mathrm{P}_{0}$. The final multiplication result is available at $\mathrm{S}_{7} \mathrm{~S}_{6} \ldots . . \mathrm{S}_{1} \mathrm{~S}_{0}$. 

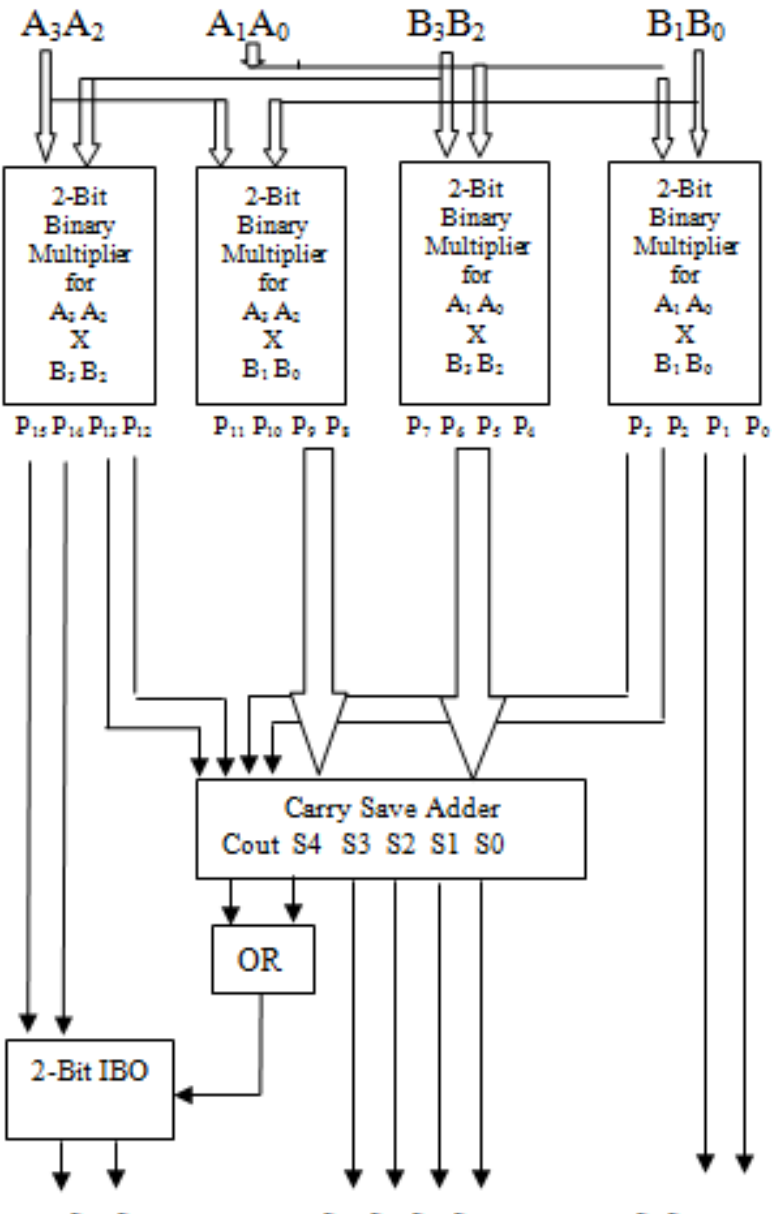
$\mathrm{S}_{7} \mathrm{~S}_{6}$
$\begin{array}{llllll}\mathrm{S}_{5} & \mathrm{~S}_{4} & \mathrm{~S}_{3} & \mathrm{~S}_{2}\end{array}$
$\mathrm{S}_{1} \mathrm{~S}_{0}$

Fig. 4. Generalized block diagram for 4-bit modified Vedic multiplier

As shown in Fig. 2, the divided parts are of 4-bit each for an 8-bit multiplication. The mod-7 calculator gives 3-bit data because the values are less than 7 . The output of mod-7 needs to be multiplied, therefore $\mathrm{A}_{3}$ and $\mathrm{B}_{3}$ are always ' 0 ' for the last input bit of each data of the 4-bit Vedic multiplier. The multiplication operation between $A_{3} A_{2}$ and $B_{3} B_{2}$ is reduced to ANDing of $\mathrm{A}_{2}$ and $\mathrm{B}_{2}$ at $\mathrm{P}_{12}$ and the terms $\mathrm{P}_{13}, \mathrm{P}_{14}$ and $\mathrm{P}_{15}$ are ' 0 '. Moreover, the multiplication output from each Vedic multiplier block is limited to the maximum value of 36, so the result can be accommodated in $\mathrm{S}_{5} \mathrm{~S}_{4} \mathrm{~S}_{3} \mathrm{~S}_{2} \mathrm{~S}_{1} \mathrm{~S}_{0}$. Therefore, the OR gate and 2-bit IBO circuit can be removed. Similarly, the requirements for hardware optimization can be analyzed for other modulo numbers. As depicted in Fig. 2, the extracted 6-bit output from all the four Vedic multipliers is provided to mod-7 calculator. It produces $\mathrm{P}_{3}, \mathrm{P}_{2}, \mathrm{P}_{1}$ and $\mathrm{P}_{0}$ corresponding to mod-7 value. The value of $\mathrm{P}_{1}$ and $\mathrm{P}_{2}$ is added using a mod-7 adder, shifted left by 1-bit and then again passed through a mod-7 calculator. The obtained output is represented as $\mathrm{P}_{5}$. Similarly, the value of $\mathrm{P}_{3}$ is shifted left by 2-bit and then mod-7 is computed and represented as $\mathrm{P}_{4}$. Finally, the values of $\mathrm{P}_{0}, \mathrm{P}_{4}$ and $\mathrm{P}_{5}$ are added using a mod-7 adder to obtain the modulo multiplication result of $|\mathrm{X} . \mathrm{Y}|_{\mathrm{m}}$. The proposed design is implemented in VHDL and synthesized using the Virtex-4 ML-402 board from Xilinx ISE 14.1.

\section{DESIGN STRATEGIES FOR RNS VEDIC-BASED MULTIPLIER FOR DIFFERENT MODULI}

The design proposed in Section II can easily be extended for larger input data sizes of multipliers (as a power of 2), such as $N=16$ and $N=32$. When $N=16$, the subdivided parts will have the data size of 8-bit and the LUT should have eight address lines. The details of address and corresponding stored value in LUT are presented in Table III for 8-bit input data.

Table III. LUT contents for mod-7 for 8-bit data size

\begin{tabular}{|c|c|}
\hline LUT address & LUT contents \\
\hline 000 & 001 \\
\hline 001 & 010 \\
\hline 010 & 100 \\
\hline 011 & 001 \\
\hline 100 & 010 \\
\hline 101 & 100 \\
\hline 110 & 001 \\
\hline 111 & 010 \\
\hline
\end{tabular}

The multiplier design proposed for $\mathrm{m}=7$ in Fig. 2 needs to be modified to work for other modulo numbers. The suggested modifications are shown in Table IV for different values of $\mathrm{m}$ and $\mathrm{N}$.

Table IV. Suggested Modifications In The Proposed Design For Other Modulo Numbers

\begin{tabular}{|c|c|l|}
\hline $\begin{array}{c}\text { Mod } \\
(\mathrm{m})\end{array}$ & \multicolumn{1}{|c|}{$\begin{array}{c}\text { Input data } \\
\text { size (N) }\end{array}$} & \multicolumn{1}{c|}{ Hardware requirements } \\
\hline 3 & $\mathrm{~N}=4,8,16,32$ & $\begin{array}{l}\text { 2-bit multiplier, no need of } \\
\text { shifting in middle and left stages } \\
\text { w.r.t. Fig. 2 }\end{array}$ \\
\hline 5 & $\mathrm{~N}=8,16,32$ & $\begin{array}{l}\text { 2-bit multiplier, needs shifting } \\
\text { left by 2-bit in middle stage and } \\
\text { no shifting in left stage w.r.t. Fig. } \\
2\end{array}$ \\
\cline { 2 - 3 } & $\mathrm{N}=4$ & $\begin{array}{l}\text { Vedic multiplier of 4-bit, no need } \\
\text { of shifting in middle and left } \\
\text { stages w.r.t. Fig. 2 }\end{array}$ \\
\hline 7 & $\begin{array}{l}\text { Vedic multiplier of 4-bit, needs } \\
\text { shifting left by 1-bit in middle } \\
\text { stage and 2-bit in left stage w.r.t. } \\
\text { Fig. 2 }\end{array}$ \\
\hline & $\begin{array}{l}\text { Needs shifting left by 2-bit in } \\
\text { middle stage and 1-bit in left } \\
\text { stage w.r.t. Fig. 2 }\end{array}$ \\
\hline
\end{tabular}

The simulation results for the mod-7 modulo multiplier for $\mathrm{N}=8,16$ and 32 are exhibited in Fig. 5. The design is tested by using different set of input data. The input and output signal values are represented in integer inside the waveform window for clarity. 

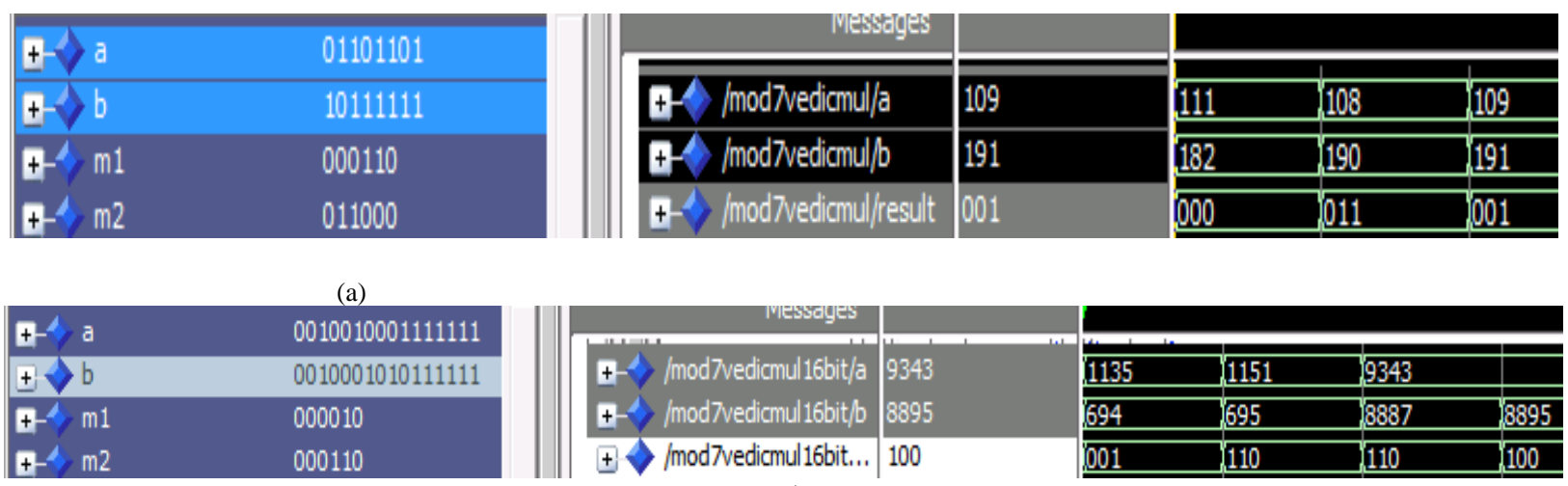

(b)

\begin{tabular}{ll} 
\pm+ a & 000010001011001101 \\
$\pm-b$ & 000000110000001111 \\
\hline$+m 1$ & 000000 \\
$+-m 2$ & 000000
\end{tabular}

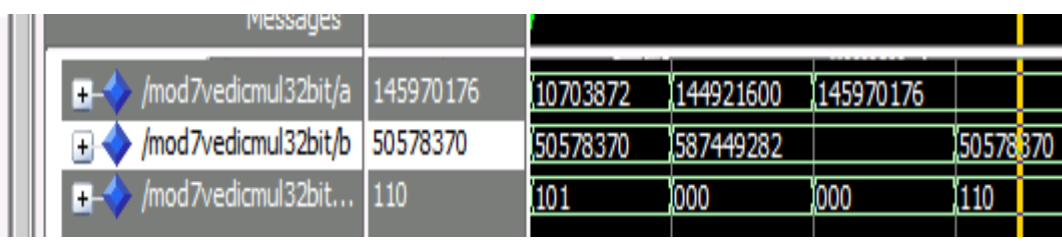

(c)

Fig. 5. Simulation results of modulo multiplier for $m=7:$ (a) $N=8$, (b) $N=16$ and (c) $N=32$.

\section{COMPARATIVE ANALYSIS}

The binary adder is used as a fundamental building block in all the designs, including modulo adder, modulo calculator and Vedic multiplier. The basic ripple carry adder and carry look-ahead adder circuits are used in this work to compare the modulo multiplier architectures. Nonetheless, the synthesis results can be improved by using the advanced adders reported in [8]-[13]. The synthesis results of the proposed multiplication technique are compared with the direct computation using the Xilinx Virtex-4 ML-402 FPGA board. For direct modulo multiplication, the Vedic multiplier is used followed by the respective mod-7 calculator. In the work presented, this method is termed as Direct-I. In addition, the computation of $|\mathrm{A} . \mathrm{B}|_{\mathrm{m}}$ can be done by first computing $|\mathrm{A}|_{\mathrm{m}}$ and $|\mathrm{B}|_{\mathrm{m}}$ and then computing mod-m of the product of $|\mathrm{A}|_{\mathrm{m}}$ and $|\mathrm{B}|_{\mathrm{m}}$. This method is termed as Direct-II.

Table V. Comparison Of Modulo Multipliers For Mod-7.

\begin{tabular}{|c|c|c|c|c|}
\hline $\begin{array}{c}\text { Input data } \\
\text { size (N) }\end{array}$ & Technique & $\begin{array}{c}\text { No. of } \\
\text { slices }\end{array}$ & $\begin{array}{c}\text { No. of } \\
\text { LUTs }\end{array}$ & $\begin{array}{c}\text { Delay } \\
\text { (ns) }\end{array}$ \\
\hline \multirow{3}{*}{8} & Proposed & 79 & 143 & 12.96 \\
& Direct-I & 113 & 202 & 15.63 \\
& Direct-II & 59 & 106 & 16.02 \\
\hline \multirow{3}{*}{16} & Proposed & 167 & 299 & 15.51 \\
& Direct-I & 486 & 856 & 28.58 \\
& Direct-II & 54 & 104 & 22.82 \\
& & & & \\
\hline \multirow{3}{*}{32} & Proposed & 151 & 287 & 18.81 \\
& Direct-I & 1905 & 3341 & 53.78 \\
& Direct-II & 137 & 262 & 27.08 \\
& & & & \\
\hline
\end{tabular}

The performance comparison has also been done in terms of area, delay and power using the $32 \mathrm{~nm}$ standard cell library in Synopsys Design Compiler. Table VI presents the comparative analysis for the high voltage threshold (HVT) library.
Table VI. Performance Comparison Using the $32 \mathrm{Nm}$ HVT Standard Cell Library

\begin{tabular}{|c|c|c|c|c|}
\hline $\begin{array}{c}\text { Input data } \\
\text { size (N) }\end{array}$ & Technique & $\begin{array}{c}\text { Area } \\
\left(\mu \mathrm{m}^{2}\right)\end{array}$ & $\begin{array}{c}\text { Delay } \\
(\mathrm{ns})\end{array}$ & $\begin{array}{c}\text { Power } \\
(\mu \mathrm{W})\end{array}$ \\
\hline \multirow{3}{*}{8} & Proposed & 6802 & 6.14 & 280 \\
& Direct-I & 3075 & 7.22 & 188 \\
& Direct-II & 2717 & 5.96 & 131 \\
\hline \multirow{3}{*}{16} & Proposed & 8607 & 7.99 & 470 \\
& Direct-I & 9452 & 14.7 & 655 \\
& Direct-II & 4526 & 9.71 & 285 \\
\hline \multirow{3}{*}{32} & Proposed & 12242 & 11.75 & 830 \\
& Direct-I & 33088 & 29.67 & 2212 \\
& Direct-II & 8156 & 17.19 & 670 \\
\hline
\end{tabular}

It can be observed that as the input data size increases, the proposed methodology for the modular multiplication is more efficient than the direct computation methods.

\section{CONCLUSION}

An efficient modulo multiplier based on Vedic mathmatics is proposed in this paper. A detailed design analysis is presented for mod-7 modulo multiplier for the input data sizes of 8,16 and 32 bits. The design process can be extended for other modulo values after making the modifications suggested in Table IV. The proposed design is compared with direct computation, and the comparison results are presented in Tables V and VI. It can be inferred that the proposed design is efficient in terms of delay when the input data size is large, irrespective of the design plateforms, such as FPGA and standard cell. Furthermore, the use of different architectures of adders, including carry save adder, square root carry select adder and modified square root carry select adder, may be proposed for future work.

\section{REFERENCES}

1. S. Akhter, "VHDL implementation of fast $\mathrm{N} \times \mathrm{N}$ multiplier based on Vedic mathematics," in Proc. 18th European Conference on Circuit Theory and Design, 2007, pp. 472-475. 
2. S. Akhter and S. Chaturvedi, "Modified binary multiplier circuit based on Vedic mathematics," in Proc. IEEE 2019 International Conference on Signal Processing and Integrated Networks, 2019, pp. 234-237.

3. A. Omondi and B. Premkumar, Residue number systems: Theory and implementation, 1st ed. London: Imperial College Press, 2007.

4. A. A. Hiasat, "General modular adder designs for residue number system applications," IET Circuits, Devices \& Systems, vol. 12, no. 4, pp. 424-431, Aug. 2018.

5. A. A. Hiasat, "High-speed and reduced-area modular adder structures for RNS," IEEE Transactions on Computers, vol. 51, no. 1, pp. 84-89, Jan. 2002.

6. S. Akhter, G. Raturi, and S. Khan, "Analysis and design of residue number system based building blocks," in Proc. IEEE 2018 International Conference on Signal Processing and Integrated Networks, 2018, pp. 441-445.

7. T. Gupta, S. Akhter, A. Srivastava, and S. Chaturvedi, "HDL implementation of five moduli residue number system," International Journal of Innovative Technology and Exploring Engineering, vol. 8, no. 9, pp. 689-693, Jul. 2019.

8. S. Akhter, S. Chaturvedi, and K. Pardhasardi, "CMOS implementation of efficient 16-bit square root carry-select adder," in Proc. IEEE 2015 International Conference on Signal Processing and Integrated Networks, 2015, pp. 891-896.

9. B. Ramkumar and H. M. Kittur, "Low-power and area-efficient carry select adder," IEEE Transactions on Very Large Scale Integration Systems, vol. 20, no. 2, pp. 371-375, Feb. 2012.

10.G. Singh, "Design of low area and low power modified 32-bit square roo carry select adder," International Journal of Engineering Research and General Science, vol. 2, no. 4, pp. 422-431, Jun. 2014.

11.Y. He, C.-H. Chang, and J. Gu "An area efficient 64-bit square root carry-select adder for low power applications," in Proc. IEEE 2005 International Symposium on Circuits and Systems, 2005, pp. 4082-4085.

12.V. Kokilavani, K. Preethi, and P. Balasubramanian, "FPGA-based synthesis of high-speed hybrid carry select adders," Advances in Electronics, vol. 2015, pp. 1-13, 2015.

13.S. Akhter, V. Saini, and J. Saini, "Analysis of Vedic multiplier using various adder topologies," in Proc. IEEE 2017 International Conference on Signal Processing and Integrated Networks, 2017, pp. 173-176.

\section{AUTHORS PROFILE}

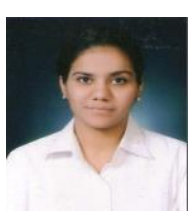

Tukur Gupta received her B.Tech. in Electronics and Communication Engineering (ECE) from the Gautam Buddha Technical University (Formerly, Uttar Pradesh Technical University, Lucknow) in 2010 and M.Tech. in Microelectronics and Embedded Technology from Jaypee Institute of Information Technology (JIIT), NOIDA in 2015. She is currently pursuing Ph.D. in VLSI Design from JIIT, NOIDA. She has 6 years of industrial and teaching experience. Her research interests include VLSI design and low-power design.

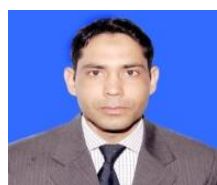

Shaheen Khan obtained his

B.Tech. and M.Tech. degrees in ECE from ITM, Gurgaon, India and RV University, Udaipur, India in 2001 and 2006, respectively. He is currently pursuing his Ph.D. degree from Jamia Millia Islamia, New Delhi, India. His research area includes digital system design and signal processing. He is a Life Member of IETE.

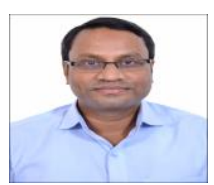

Shamim Akhter did B.Tech. (ECE) from AMU, Aligarh (2001), M.Tech. (VLSI) from IIT Delhi (2003) and Ph.D. from JIIT NOIDA (2015). His research interest is VLSI signal processing.

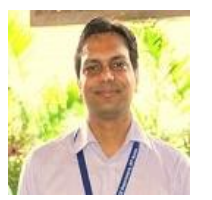

Saurabh Chaturvedi obtained his B.Tech. degree in ECE from JIIT, NOIDA in 2005, M.Tech. degree in VLSI Design from the Guru Gobind Singh Indraprastha University, Delhi, India in 2008 and Ph.D. degree from the University of Johannesburg, South Africa in 2018. He is a Senior Member of IEEE. 